\title{
Classification of modules of the intermediate series over Ramond $N=2$ superconformal algebras 1
}

\author{
Jiayuan Fu \\ Department of Mathematics, Communication University of China \\ Beijing 100024, China \\ Email: fujy@cuc.edu.cn \\ Qifen Jiang \\ Department of Mathematics, Shanghai Jiaotong University, Shanghai 200240, China \\ Email: qfjiang@sjtu.edu.cn \\ Yucai Su \\ Department of Mathematics, University of Science and Technology of China \\ Anhui 230026, China \\ Email: ycsu@ustc.edu.cn
}

\begin{abstract}
In this paper, we first discuss the structure of the Ramond $N=2$ superconformal algebras. Then we classify the modules of the intermediate series over Ramond $N=2$ superconformal algebra.
\end{abstract}

\section{Introduction}

More than two decades ago, superconformal algebras were first constructed independently and almost at the same time by Kac [4] and by Ademollo et al. [1]. On the mathematical side Kac and van de Leuer [5], Cheng and Kac [3] have classified all possible superconformal algebras and Kac recently has proved that their classification is complete.

The Neveu-Schwarz, the Ramond and the Topological $N=2$ superconformal algebras are connected to each other by the spectral flows and/or the topological twists. Therefore, we only consider the Ramond $N=2$ superconformal algebra, which is a $\mathbb{Z}_{2}$-graded space: $\mathcal{L}=\mathcal{L}_{\overline{0}} \oplus \mathcal{L}_{\overline{1}}$, with

$$
\mathcal{L}_{\overline{0}}=\operatorname{span}_{\mathbb{C}}\left\{L_{i}, H_{j}, c \mid i, j \in \mathbb{Z}\right\}, \quad \mathcal{L}_{\overline{1}}=\operatorname{span}_{\mathbb{C}}\left\{G_{i}^{-}, G_{j}^{+} \mid i, j \in \mathbb{Z}\right\},
$$

such that $c$ is a central element and the following relations hold:

$$
\begin{array}{ll}
{\left[L_{i}, L_{j}\right]=(i-j) L_{i+j}+\frac{1}{12}\left(i^{3}-i\right) \delta_{i+j, 0} c} \\
{\left[L_{i}, H_{j}\right]=-j H_{i+j}, \quad\left[H_{i}, H_{j}\right]=\frac{1}{3} i \delta_{i+j, 0} c} \\
{\left[L_{i}, G_{j}^{ \pm}\right]=\left(\frac{i}{2}-j\right) G_{i+j}^{ \pm}, \quad\left[H_{i}, G_{j}^{ \pm}\right]= \pm G_{i+j}^{ \pm},} \\
{\left[G_{i}^{+}, G_{j}^{+}\right]=\left[G_{i}^{-}, G_{j}^{-}\right]=0, \quad\left[G_{i}^{-}, G_{j}^{+}\right]=2 L_{i+j}-(i-j) H_{i+j}+\frac{1}{3}\left(i^{2}-\frac{1}{4}\right) \delta_{i+j, 0} c .}
\end{array}
$$

Obviously, the Cartan subalgebra of $\mathcal{L}$ is $\mathcal{H}=\mathbb{C} L_{0}+\mathbb{C} H_{0}+\mathbb{C} c$, and Vir = $\operatorname{span}_{\mathbb{C}}\left\{L_{m}, c \mid m \in \mathbb{Z}\right\}$ is a Virasoro subalgebra of $\mathcal{L}$, which can be described as the

\footnotetext{
${ }^{1}$ Supported by Communication University of China, NSF grants 10571120, 10471096 of China and "One Hundred Talents Program" from University of Science and Technology of China

Correspondence author: J. Fu: fujy@cuc.edu.cn
} 
universal central extensions of the Lie algebras of differential operators (see [2] for details).

An $\mathcal{L}$-module $V$ is called a Harish-Chandra module if $V$ is a direct sum of its finite dimensional weight spaces $V^{\lambda}=\{v \in V \mid x \cdot v=\lambda(x) v, x \in \mathcal{H}\}$ for all $\lambda \in \mathcal{H}^{*}$ (the dual of $\mathcal{H}$ ). Similar to the case of Virasoro algebra, we can define the module of the intermediate series over $\mathcal{L}$ :

Definition 1.1 A module of the intermediate series over $\mathcal{L}$ is an indecomposable Harish-Chandra module $V$ such that $\operatorname{dim} V^{\lambda} \leqslant 1$ for all $\lambda \in \mathcal{H}^{*}$.

In this paper, we will consider some properties of $\mathcal{L}$, basing on representations of the above type over the Virasoro algebra.

The paper is arranged as follows. In Section 2, we first consider all possible super-extensions of the Heisenberg-Virasoro type Lie algebra. Our main result in this section is Theorem 2.1. As a conclusion, we obtain that the Ramond $N=2$ superconformal algebra is a special case of such super-extension. Then we study the modules of the intermediate series in the last section.

Our main result is

Theorem 1.2. Any indecomposable module of the intermediate series $V$ over the Ramond $N=2$ superconformal algebra is one of modules $R A_{a, b}, R A_{\alpha}, R A^{\beta}, R B_{a, b}$, $R B_{\alpha}, R B^{\beta}$, or one of their quotients for $a, b, \alpha, \beta \in \mathbb{C}$, where $R A_{a, b}$ is defined in (3.25), $R B_{a, b}$ is defined in (3.26),$R A_{\alpha}$ is defined in (3.39), $R A^{\beta}$ is defined in (3.40), (3.41), $R B_{\alpha}$ is defined in (3.42), $R B^{\beta}$ is defined in (3.43).

\section{The structure of the Ramond $N=2$ superconformal algebras}

Let $\tilde{\mathcal{L}}_{\overline{0}}=\operatorname{span}_{\mathbb{C}}\left\{L_{m}, H_{n} \mid m, n \in \mathbb{Z}\right\}$ be a Heisenberg-Virasoro type algebra (only with 1-dimensional center $\mathbb{C} H_{0}$ ) with the following Lie brackets:

$$
\left[L_{m}, L_{n}\right]=(m-n) L_{m+n}, \quad\left[H_{m}, H_{n}\right]=0, \quad\left[L_{m}, H_{n}\right]=-n H_{m+n} .
$$

Let us consider all possible super-extensions of the Lie algebra $\tilde{\mathcal{L}}_{\overline{0}}$. First assume that $\tilde{\mathcal{L}}_{\overline{1}}$ is an $\tilde{\mathcal{L}}_{\overline{0}}$-module of intermediate series with basis $\left\{G_{i} \mid i \in \mathbb{Z}\right\}$ such that $\tilde{\mathcal{L}}_{\overline{0}} \oplus \tilde{\mathcal{L}}_{\overline{1}}$ is a Lie superalgebra. Then following [8, Theorem 3.2], we can suppose

$$
\left[L_{m}, G_{i}\right]=(a-i+m b) G_{m+i}, \quad\left[H_{n}, G_{i}\right]=f G_{m+i},
$$

for some $a, b, f \in \mathbb{C}, f \neq 0$. Set $\left[G_{i}, G_{j}\right]=a_{i j} L_{i+j}+b_{i j} H_{i+j}$, for some $a_{i j}, b_{i j} \in \mathbb{C}$. Then from the equation

$$
\left[H_{k},\left[G_{i}, G_{j}\right]\right]=\left[\left[H_{k}, G_{i}\right], G_{j}\right]+\left[G_{i},\left[H_{k}, G_{j}\right]\right],
$$

by letting $k=0$, we deduce $2 f\left[G_{i}, G_{j}\right]=0$. Therefore, $\left[G_{i}, G_{j}\right]=0$ for all $i, j \in \mathbb{Z}$. This is a trivial extension and not the thing we are interested in. Hence we suppose that $\tilde{\mathcal{L}}_{\overline{1}}$ is a direct sum of two $\tilde{\mathcal{L}}_{\overline{0}}$-modules of intermediate series, with basis $\left\{G_{i}^{ \pm} \mid\right.$ $i \in \mathbb{Z}\}$. Then we have the following equations:

$$
\left[L_{m}, G_{i}^{+}\right]=\left(a^{+}-i+m b^{+}\right) G_{m+i}^{+}, \quad\left[H_{n}, G_{i}^{+}\right]=f_{1} G_{m+i}^{+},
$$




$$
\left[L_{m}, G_{i}^{-}\right]=\left(a^{-}-i+m b^{-}\right) G_{m+i}^{-}, \quad\left[H_{n}, G_{i}^{-}\right]=f_{2} G_{m+i}^{-},
$$

where $a^{+}, a^{-}, b^{+}, b^{-}, f_{1}, f_{2} \in \mathbb{C}$, and $\left(f_{1}, f_{2}\right) \neq(0,0)$. In order to get a nontrivial super-extension, we must have $a^{+}=a^{-}$, denoted by $a$. Set

$$
\left[G_{i}^{-}, G_{j}^{+}\right]=a_{i j} L_{i+j}+b_{i j} H_{i+j} .
$$

Since

$$
0=\left[H_{0},\left[G_{i}^{-}, G_{j}^{+}\right]\right]=\left(f_{1}+f_{2}\right)\left[G_{i}^{-}, G_{j}^{+}\right],
$$

we can suppose that $f_{1}=1, f_{2}=-1$ (replacing $H_{m}$ by $f_{1}^{-1} H_{m}$ ). Following

$$
\left[L_{k},\left[G_{i}^{-}, G_{j}^{+}\right]\right]=\left[\left[L_{k}, G_{i}^{-}\right], G_{j}^{+}\right]+\left[G_{i}^{-},\left[L_{k}, G_{j}^{+}\right]\right],
$$

and setting $k=0$ in (2.2), we can get that $a=0$. Following (2.2), we have

$$
\begin{aligned}
(k-i-j) a_{i, j} & =\left(-i+k b^{-}\right) a_{i+k, j}+\left(-j+k b^{+}\right) a_{i, k+j}, \\
(-i-j) b_{i, j} & =\left(-i+k b^{-}\right) b_{i+k, j}+\left(-j+k b^{+}\right) b_{i, k+j} .
\end{aligned}
$$

By

$$
\left[G_{k}^{-},\left[G_{i}^{-}, G_{j}^{+}\right]\right]+\left[G_{i}^{-},\left[G_{k}^{-}, G_{j}^{+}\right]\right]=0,
$$

we obtain $b_{i j}+b_{k j}=a_{i j}\left(-k+(i+j) b^{-}\right)+a_{k j}\left(-i+(k+j) b^{-}\right)$. Setting $k=i$ gives

$$
b_{i j}=a_{i j}\left(-i+(i+j) b^{-}\right) .
$$

Replacing $G_{k}^{-}, G_{i}^{-}, G_{j}^{+}$respectively by $G_{j}^{+}, G_{j}^{+}, G_{i}^{-}$in (2.5) gives

$$
b_{i j}=-a_{i j}\left(-j+(i+j) b^{+}\right) .
$$

Comparing it with (2.6), we can get that

$$
b^{+}+b^{-}=1 .
$$

Following $\left[H_{k},\left[G_{i}^{-}, G_{j}^{+}\right]\right]=\left[\left[H_{k}, G_{i}^{-}\right], G_{j}^{+}\right]+\left[G_{i}^{-},\left[H_{k}, G_{j}^{+}\right]\right]$, we have that

$$
a_{k+i, j}=a_{i, k+j}, \quad k a_{i, j}=b_{i, k+j}-b_{k+i, j} \quad \text { for all } i, j, k \in \mathbb{Z} .
$$

Letting $i=0, k=i$ gives

$$
a_{i, j}=a_{0, i+j} \text { for all } i, j \in \mathbb{Z} .
$$

Taking $i=0$ in (2.3), and by (2.7), we obtain that

$$
(k-j) a_{0, j}=k b^{-} a_{k, j}+\left(-j+k b^{+}\right) a_{0, k+j}=(k-j) a_{0, k+j} .
$$

Let $a_{0,0}=d \in \mathbb{C}$, then by (2.77) and (2.8), we have that $a_{i j}=d$ for all $i, j \in \mathbb{Z}$. Since $b^{+}+b^{-}=1$, we set $b^{-}=b$, then

$$
b^{+}=1-b, \text { and } b_{i j}=d(-i+(i+j) b) .
$$


We can set $d=1$ (replace $G_{i}^{ \pm}$by $\frac{1}{\sqrt{d}} G_{i}^{ \pm}$), then $\tilde{\mathcal{L}}=\tilde{\mathcal{L}}_{\overline{0}} \oplus \tilde{\mathcal{L}}_{\overline{1}}$ is a superalgebra with (2.1) and the following Lie brackets:

$$
\begin{array}{ll}
{\left[L_{m}, G_{n}^{+}\right]=(-n+m(1-b)) G_{m+n}^{+},} & {\left[L_{m}, G_{n}^{-}\right]=(-n+m b) G_{m+n}^{-},} \\
{\left[H_{m}, G_{n}^{ \pm}\right]= \pm G_{m+n}^{ \pm},} & {\left[G_{m}^{ \pm}, G_{n}^{ \pm}\right]=0} \\
{\left[G_{m}^{-}, G_{n}^{+}\right]=L_{m+n}+(-m+(m+n) b) H_{m+n}}
\end{array}
$$

where $m, n \in \mathbb{Z}$. Obviously, $\tilde{\mathcal{L}}$ is $\mathbb{Z}$-graded:

$$
\tilde{\mathcal{L}}=\bigoplus_{n \in \mathbb{Z}} \tilde{\mathcal{L}}_{n}, \quad \tilde{\mathcal{L}}_{n}=\left\{x \in \tilde{\mathcal{L}} \mid\left[L_{0}, x\right]=n x\right\}=\operatorname{span}_{\mathbb{C}}\left\{L_{-n}, H_{-n}, G_{-n}^{ \pm}\right\} .
$$

Now we consider the central extension of $\tilde{\mathcal{L}}$. Suppose $\varphi: \tilde{\mathcal{L}} \times \tilde{\mathcal{L}} \rightarrow \mathbb{C}$ is a 2-cocycle of $\tilde{\mathcal{L}}$, we define a linear map $f: \tilde{\mathcal{L}} \rightarrow \mathbb{C}$ as follows:

$$
\begin{array}{ll}
f\left(\tilde{\mathcal{L}}_{i}\right)=\frac{1}{i} \varphi\left(L_{0}, \tilde{\mathcal{L}}_{i}\right), \quad i \neq 0, & f\left(L_{0}\right)=\frac{1}{2} \varphi\left(L_{1}, L_{-1}\right), \\
f\left(H_{0}\right)=\varphi\left(L_{1}, H_{-1}\right), & f\left(G_{0}^{ \pm}\right)= \pm \varphi\left(H_{1}, G_{-1}^{ \pm}\right) .
\end{array}
$$

If we define another 2-cocycle of $\tilde{\mathcal{L}}, \psi: \tilde{\mathcal{L}} \times \tilde{\mathcal{L}} \rightarrow \mathbb{C}$ satisfying $\psi=\varphi-\varphi_{f}$, where $\varphi_{f}(x, y)=f([x, y])$, then

$$
\begin{aligned}
\psi\left(L_{0}, \tilde{\mathcal{L}}_{i}\right) & =\varphi\left(L_{0}, \tilde{\mathcal{L}}_{i}\right)-\varphi_{f}\left(L_{0}, \tilde{\mathcal{L}}_{i}\right)=0, \quad i \neq 0, \\
2 \psi\left(L_{0}, L_{0}\right) & =\psi\left(\left[L_{1}, L_{-1}\right], L_{0}\right)=0,
\end{aligned}
$$

Similarly, we have that $\psi\left(L_{0}, H_{0}\right)=\psi\left(L_{0}, G_{0}^{ \pm}\right)=0$. Thus

$$
\psi\left(L_{0}, \tilde{\mathcal{L}}_{i}\right)=0 \quad \text { for all } i \in \mathbb{Z} \text {. }
$$

Furthermore,

$$
i \psi\left(\tilde{\mathcal{L}}_{i}, \tilde{\mathcal{L}}_{j}\right)=\psi\left(\left[L_{0}, \tilde{\mathcal{L}}_{i}\right], \tilde{\mathcal{L}}_{j}\right)=\psi\left(L_{0},\left[\tilde{\mathcal{L}}_{i}, \tilde{\mathcal{L}}_{j}\right]\right)-\psi\left(\tilde{\mathcal{L}}_{i},\left[L_{0}, \tilde{\mathcal{L}}_{j}\right]\right)=-j \psi\left(\tilde{\mathcal{L}}_{i}, \tilde{\mathcal{L}}_{j}\right)
$$

therefore,

$$
\psi\left(\tilde{\mathcal{L}}_{i}, \tilde{\mathcal{L}}_{j}\right)=0 \quad \text { if } i+j \neq 0 .
$$

Now let us consider $\psi\left(L_{i}, L_{-i}\right)$. It follows (2.10) that

$$
\psi\left(L_{1}, L_{-1}\right)=\varphi\left(L_{1}, L_{-1}\right)-\varphi_{f}\left(L_{1}, L_{-1}\right)=0 .
$$

Then

$$
(i-2) \psi\left(L_{i}, L_{-i}\right)=\psi\left(\left[L_{i-1}, L_{1}\right], L_{-i}\right)=(i+1) \psi\left(L_{i-1}, L_{-i+1}\right) .
$$

Set $\psi\left(L_{i}, L_{-i}\right)=l_{i}$, we obtain $l_{i}=\frac{i+1}{i-2} l_{i-1}$ for $i \neq 2$, i.e.,

$$
l_{i}=\frac{i^{3}-i}{6} l_{2} \quad \text { for all } i \geqslant 3 \text {. }
$$


Then we can rewrite $\psi\left(L_{i}, L_{-i}\right)$ as follows

$$
\psi\left(L_{i}, L_{-i}\right)=\frac{i^{3}-i}{6} c_{L} \quad \text { for all } i \in \mathbb{Z}
$$

where $c_{L} \in \mathbb{C}$. Similar to the argument about $\psi\left(L_{i}, L_{-i}\right)$, we can obtain that

$$
\begin{aligned}
& \psi\left(H_{i}, H_{-i}\right)=i c_{H}, \quad \psi\left(L_{i}, H_{-i}\right)=\frac{i(i-1)}{2} c_{H L}, \\
& \psi\left(H_{i}, G_{-i}^{ \pm}\right)=\psi\left(L_{i}, G_{-i}^{ \pm}\right)=\psi\left(G_{i}^{ \pm}, G_{-i}^{ \pm}\right)=0 \quad \text { for all } i \in \mathbb{Z}
\end{aligned}
$$

where $c_{H}, c_{H L} \in \mathbb{C}$. Finally, we consider $\psi\left(G_{i}^{-}, G_{-i}^{+}\right)$. Set $\psi\left(G_{0}^{-}, G_{0}^{+}\right)=c_{G}$, by (2.9) and (2.14), we have

$$
\begin{aligned}
\psi\left(G_{1}^{-}, G_{-1}^{+}\right) & =\psi\left(\left[G_{0}^{-}, H_{1}\right], G_{-1}^{+}\right) \\
& =\psi\left(G_{0}^{-},\left[H_{1}, G_{-1}^{+}\right]\right)-\psi\left(H_{1},\left[G_{0}^{-}, G_{-1}^{+}\right]\right)=c_{G}+c_{H L}+b c_{H},
\end{aligned}
$$

and $\psi\left(G_{1}^{-}, G_{-1}^{+}\right)=\psi\left(G_{1}^{-},\left[H_{-1}, G_{0}^{+}\right]\right)=c_{G}+(1-b) c_{H}$. Hence $c_{G}+c_{H L}+b c_{H}=$ $c_{G}+(1-b) c_{H}$, i.e.,

$$
c_{H L}=(1-2 b) c_{H}
$$

Then

$$
\begin{aligned}
\psi\left(G_{i}^{-}, G_{-i}^{+}\right) & =\psi\left(\left[G_{0}^{-}, H_{i}\right], G_{-i}^{+}\right) \\
& =\psi\left(G_{0}^{-},\left[H_{i}, G_{-i}^{+}\right]\right)-\psi\left(H_{i},\left[G_{0}^{-}, G_{-i}^{+}\right]\right)=c_{G}+\left(\frac{i(i+1)}{2}-i b\right) c_{H}
\end{aligned}
$$

Note that

$$
\left[L_{i},\left[G_{j}^{-}, G_{k}^{+}\right]\right]=\left[\left[L_{i}, G_{j}^{-}\right], G_{k}^{+}\right]+\left[G_{j}^{-},\left[L_{i}, G_{k}^{+}\right]\right] .
$$

If we suppose $i+j+k=0$, then

$$
\begin{aligned}
& \frac{i^{3}-i}{6} c_{L}+(-j+(k+j) b) \frac{i(i-1)}{2} c_{H L} \\
& =(-j+i b)\left(c_{G}+\left(\frac{-k(1-k)}{2}+k b\right) c_{H}\right)+(-k+i(1-b))\left(c_{G}+\left(\frac{j(j+1)}{2}-j b\right) c_{H}\right) .
\end{aligned}
$$

By (2.16), and setting $j=0$ in (2.18), we have that

$$
\frac{i^{2}-1}{6} c_{L}-2 c_{G}=i^{2}\left(b-b^{2}\right) c_{H} .
$$

Letting $i=1$ in (2.19), we can obtain that

$$
c_{G}=\frac{b^{2}-b}{2} c_{H}, \quad c_{L}=6\left(b-b^{2}\right) c_{H} .
$$

Then we have the following theorem:

Theorem 2.1. The possible nontrivial super-extensions of the Heisenberg-Virasoro type algebra (2.1) are the following superalgebras:

$$
\hat{\mathcal{L}}=\operatorname{span}_{\mathbb{C}}\left\{L_{i}, H_{j}, G_{k}^{ \pm}, c_{H} \mid c_{H} \in \mathbb{C}, i, j, k \in \mathbb{Z}\right\}
$$


where $c_{H}$ is a central element and the following relations hold:

$$
\begin{array}{ll}
{\left[L_{i}, L_{j}\right]=(i-j) L_{i+j}+\left(i^{3}-i\right)\left(b-b^{2}\right) c_{H} \delta_{i+j, 0},} \\
{\left[L_{i}, H_{j}\right]=-j H_{i+j}+\frac{i(i-1)}{2}(1-2 b) c_{H} \delta_{i+j, 0},} & {\left[H_{i}, H_{j}\right]=i c_{H} \delta_{i+j, 0},} \\
{\left[L_{i}, G_{j}^{+}\right]=(-j+i(1-b)) G_{i+j}^{+},} & {\left[L_{i}, G_{j}^{-}\right]=(-j+i b) G_{i+j}^{-},} \\
{\left[H_{i}, G_{j}^{ \pm}\right]= \pm G_{i+j}^{ \pm},} & {\left[G_{i}^{+}, G_{j}^{+}\right]=\left[G_{i}^{-}, G_{j}^{-}\right]=0,} \\
{\left[G_{i}^{-}, G_{j}^{+}\right]=L_{i+j}+(-i+(i+j) b) H_{i+j}+\frac{i(i+1-2 b)+b^{2}-b}{2} c_{H} \delta_{i+j, 0} .}
\end{array}
$$

If $b=\frac{1}{2}$, then $\hat{\mathcal{L}}=\mathcal{L}$. That is to say, Ramond $N=2$ superconformal algebra $\mathcal{L}$ is a special case of $\hat{\mathcal{L}}$.

\section{The modules of intermediate series over $\mathcal{L}$}

$\S 3.0$ Let $V=V_{0} \oplus V_{1}$ be any indecomposable $\mathcal{L}$-module with $\operatorname{dim} V_{\alpha}^{\lambda} \leqslant 1$ for all $\lambda \in \mathcal{H}^{*}, \alpha \in \mathbb{Z} / 2 \mathbb{Z}$, where

$$
V_{\alpha}^{\lambda}=\left\{v \in V_{\alpha} \mid L_{0} \cdot v=\lambda\left(L_{0}\right) v, H_{0} \cdot v=\lambda\left(H_{0}\right) v\right\} .
$$

We also have the following:

$$
V=\left(\underset{k \in \mathbb{Z}}{\oplus} V_{0}^{a+k}\right) \oplus\left(\underset{k \in \mathbb{Z}}{\oplus} V_{1}^{a+k}\right)
$$

One sees that $c$ acts trivially on $V$ (see, e.g., [6, 9]). So we can omit $c$ in (1.1).

Now we consider all possibilities of $V_{0}$ and $V_{1}$ case by case below. Let us recall the definition of $V i r$-modules $A_{a, b}, A(\alpha), B(\beta)$ (see [6]). They all have a basis $\left\{x_{i} \mid i \in \mathbb{Z}\right\}$ such that for $i, j \in \mathbb{Z}$,

$$
\begin{aligned}
& A_{a, b}: \quad L_{i} x_{j}=(a-j+i b) x_{i+j} . \\
& A(\alpha): \quad L_{i} x_{j}=-(i+j) x_{i+j}, j \neq 0, \quad L_{i} x_{0}=-i(1+(i+1) \alpha) x_{i} . \\
& B(\beta): \quad L_{i} x_{j}=-j x_{i+j}, i+j \neq 0, \quad L_{i} x_{-i}=i(1+(i+1) \beta) x_{0} .
\end{aligned}
$$

$\S 3.1$ Suppose both of $V_{0}, V_{1}$ have the form $A_{a, b}, a, b \in \mathbb{C}$. Then we choose a basis $\left\{x_{i} \mid i \in \mathbb{Z}\right\}$ of $V_{0}$ and a basis $\left\{y_{j} \mid j \in \mathbb{Z}\right\}$ of $V_{1}$ such that

$$
\begin{array}{rlrl}
L_{i} x_{j} & =(a-j+i b) x_{i+j}, & & L_{i} y_{j}=\left(a^{\prime}-j+i b^{\prime}\right) y_{i+j}, \\
H_{i} x_{j} & =f_{i j} x_{i+j}, & H_{i} y_{j}=f_{i j}^{\prime} y_{i+j}, \\
G_{i}^{ \pm} x_{j}=a_{i j}^{ \pm} y_{i+j}, & G_{i}^{ \pm} y_{j}=b_{i j}^{ \pm} x_{i+j},
\end{array}
$$

where $a, a^{\prime}, b, b^{\prime}, f_{i j}, f_{i j}^{\prime}, a_{i j}^{ \pm}, b_{i j}^{ \pm} \in \mathbb{C}$. We have $a=a^{\prime}$ by applying $L_{0}$ to the first formula of (3.5). By (1.1), we have

$$
\left(\frac{i}{2}-j\right) a_{i+j, k}^{ \pm}=\left(a-(k+j)+i b^{\prime}\right) a_{j, k}^{ \pm}-(a-k+i b) a_{j, i+k}^{ \pm},
$$


and $2(a-k+(i+j) b)-(i-j) f_{i+j, k}=a_{j, k}^{+} b_{i, k+j}^{-}+a_{i, k}^{-} b_{j, k+i}^{+}$. Let $i=j$, we get

$$
a_{i, k}^{+} b_{i, k+i}^{-}+a_{i, k}^{-} b_{i, k+i}^{+}=2(a-k+2 i b) .
$$

From (3.7), we know that for all fixed $i, k \in \mathbb{Z}$,

$$
a_{i, j}^{+}=a_{i, j}^{-}=0, b_{k, l}^{+}=b_{k, l}^{-}=0 \text { only for finitely many } j \text { and finitely many } l .
$$

Applying $\left[G_{i}^{ \pm}, G_{j}^{ \pm}\right]=0$ to $x_{k}$ gives $a_{i, j}^{ \pm} b_{i, k+j}^{ \pm}+a_{i, k}^{ \pm} b_{j, k+i}^{ \pm}=0$. Letting $i=j$ gives

$$
a_{i, k}^{ \pm} b_{i, k+i}^{ \pm}=0 .
$$

Therefore, by (3.7) and (3.9), for any $i, k \in \mathbb{Z}$ with $a-k+2 i b \neq 0$, we have

$$
a_{i k}^{+} a_{i k}^{-}=0, \quad\left(a_{i k}^{+}\right)^{2}+\left(a_{i k}^{-}\right)^{2} \neq 0, \quad\left(\text { similar relations for } b_{i k}^{ \pm}\right) .
$$

For convenience, we omit the superscript " \pm " in $a_{i j}^{ \pm}$. Let $i=2 j$ and $i=-2 j$ in (3.6) respectively, we get

$$
\begin{aligned}
(a-k+2 j b) a_{j, k+2 j} & =\left(a-(k+j)+2 j b^{\prime}\right) a_{j, k}, \\
-2 j a_{-j, k} & =\left(a-(k+j)-2 j b^{\prime}\right) a_{j, k}-(a-k-2 j b) a_{j, k-2 j} .
\end{aligned}
$$

Multiplying (3.12) by $a-(k-j)+2 j b^{\prime}$ and replacing the last term by (3.11), we get

$$
\begin{aligned}
& -2 j\left(a-(k-j)+2 j b^{\prime}\right) a_{-j, k} \\
& =\left(a-(k-j)+2 j b^{\prime}\right)\left(a-(k+j)-2 j b^{\prime}\right) a_{j, k}-(a-k-2 j b)(a-(k-2 j)+2 j b) a_{j, k} \\
& =2 j\left(a-(k-j)+2 j b^{\prime}+2 j t\right) a_{j, k},
\end{aligned}
$$

where $t=b^{\prime 2}-\left(b+\frac{1}{2}\right)^{2}$. Similarly, let $j=-j, i=2 j$ and $j=-j, i=-2 j$ in (3.6) , we can obtain that

$$
2 j\left(a-(k+j)-2 j b^{\prime}\right) a_{j, k}=-2 j\left(a-(k+j)-2 j b^{\prime}-2 j t\right) a_{-j, k} .
$$

It follows that

$$
\begin{aligned}
& \left(\left(a-(k+j)-2 j b^{\prime}\right)\left(a-(k-j)+2 j b^{\prime}\right)\right. \\
& \left.-\left(\left(a-k-j-2 j b^{\prime}\right)-2 j t\right)\left(\left(a-k+j+2 j b^{\prime}\right)+2 j t\right)\right) a_{j k}=0,
\end{aligned}
$$

which gives

$$
4 j^{2} t\left(t+2 b^{\prime}+1\right) a_{j k}=0 .
$$

By (3.8), there at least exists one $k_{0}$ such that $a_{1, k_{0}}^{+} \neq 0$, or $a_{1, k_{0}}^{-} \neq 0$. (If $a_{1, k}^{ \pm}=0$ for all $k$, by letting $j=1$ in (3.6) we get $a_{i k}^{ \pm}=0$ for $i, k \in \mathbb{Z}, i \neq 2$. By letting $i=j=1$ in (3.6), we get $a_{2, k}^{ \pm}=0$.) Thus it follows from (3.13) that

$$
b^{\prime}= \pm\left(b+\frac{1}{2}\right), \quad \text { or } \quad b^{\prime}=-1 \pm\left(b+\frac{1}{2}\right)
$$


Case 1. $b^{\prime}=b+\frac{1}{2}$.

First suppose $a-k+2 b \neq 0$ for all $k \in \mathbb{Z}$. Letting $j=1$ in (3.11), we obtain (again we omit the superscript " \pm " in $x_{0}, x_{1}$ for the time being)

$$
a_{1, k}= \begin{cases}x_{0}, & k \text { is even } \\ x_{1}, & k \text { is odd }\end{cases}
$$

Let $j=1$, and let $i, k$ be odd in (3.6), then

$$
\begin{aligned}
\left(\frac{i}{2}-1\right) a_{i+1, k} & =\left(a-(k+1)+i b^{\prime}\right) a_{1, k}-(a-k+i b) a_{1, k+i} \\
& =\left(a-(k+1)+i b^{\prime}\right) x_{1}-(a-k+i b) x_{0} .
\end{aligned}
$$

By (3.11) and $b^{\prime}=b+\frac{1}{2}$, we also have

$$
\begin{aligned}
\left(\frac{i}{2}-1\right) a_{i+1, k} & =\left(\frac{i}{2}-1\right) a_{i+1, k+2(i+1)} \\
& =\left(a-(k+2(i+1)+1)+i b^{\prime}\right) x_{1}-(a-(k+2(i+1))+i b) x_{0} .
\end{aligned}
$$

Obviously, we get $x_{0}=x_{1}$. Similar to the arguments after (3.13), we have

$$
a_{i j}^{ \pm}=d_{1}^{ \pm} \quad \text { for all } i, j \in \mathbb{Z}
$$

where $d_{1}^{ \pm}$are constants, and by (3.10),

$$
d_{1}^{+} d_{1}^{-}=0, \quad\left(d_{1}^{+}\right)^{2}+\left(d_{1}^{-}\right)^{2} \neq 0 .
$$

Now we suppose that $a-k^{\prime}+2 b=0$ for some $k^{\prime} \in \mathbb{Z}$. It follows from (3.11) that $(a-k+2 b) a_{1, k}=(a-k+2 b) a_{1, k+2}$. Then

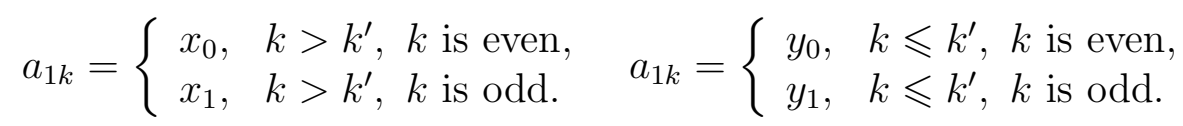

By (3.6), we get

$$
a_{i k}=\left\{\begin{array}{l}
x_{0}, \quad k>k^{\prime}, k+i-1>k^{\prime}, \text { and } k, i-1 \text { are even } \\
x_{1}, \quad k>k^{\prime}, k+i-1>k^{\prime}, \text { and } k, i-1 \text { are odd }
\end{array}\right.
$$

and

$$
a_{i k}= \begin{cases}y_{0}, & k \leqslant k^{\prime}, \quad k+i-1 \leqslant k^{\prime}, \text { and } k, i-1 \text { are even } \\ y_{1}, & k \leqslant k^{\prime}, \quad k+i-1 \leqslant k^{\prime}, \text { and } k, i-1 \text { are odd }\end{cases}
$$

Now choose some $k, j \in \mathbb{Z}$, such that $a-k+2 j b \neq 0, k \leqslant k^{\prime}, k+j-1 \leqslant k^{\prime}$, $k+2 j>k^{\prime}, k+3 j-1>k^{\prime}$, and one of $k$ and $j$ is even, and another is odd. Then by (3.11), we have

$$
(a-k+2 j b) a_{j, k}=(a-k+2 j b) a_{j, k+2 j} .
$$


Therefore,

$$
x_{0}=y_{0}, \quad x_{1}=y_{1} .
$$

Similar to the argument above, we again have (3.14) and (3.15).

Case 2. $\quad b^{\prime}=-\left(b+\frac{1}{2}\right)$.

Let $i=2 j$ and $i=-2 j$ respectively in (3.6), we have

$$
\begin{aligned}
& (a-k-2 j-2 j b) a_{j, k}=(a-k+2 j b) a_{j, k+2 j}, \\
& -2 j a_{-j, k}=(a-k+2 j b) a_{j, k}-(a-k-2 j b) a_{j, k-2 j}=-2 j a_{j, k},
\end{aligned}
$$

where the second equality of (3.17) follows from (3.16) by replaced $k$ by $k-2 j$. Hence, $a_{j, k}=a_{-j, k}$ for all $k, j \in \mathbb{Z}$. Using it, again by (3.6), we deduce that

$$
\begin{aligned}
& \left(a-(k+j)+i b^{\prime}\right) a_{j, k}-(a-k+i b) a_{j, k+i}=\left(\frac{i}{2}-j\right) a_{i+j, k} \\
& =\left(\frac{i}{2}-j\right) a_{-i-j, k}=-\left(a-(k-j)-i b^{\prime}\right) a_{-j, k}-(a-k-i b) a_{-j, k-i} .
\end{aligned}
$$

Hence

$$
(a-k-i b) a_{j, k-i}-2(a-k) a_{j, k}+(a-k+i b) a_{j, k+i}=0 .
$$

Let $j=1$ in (3.16) and then replace $k$ by $k+2$ in the new equality, we can obtain

$$
a_{1, k+4}=\frac{(a-k-4-2 b)(a-k-2-2 b)}{(a-k-2+2 b)(a-k+2 b)} a_{1, k} .
$$

Similarly, we can have a formula for $a_{1, k-4}$. Then let $i=4$ in (3.18), we have

$$
\begin{aligned}
& \left((a-k-4 b) \frac{(a-k+4+2 b)(a-k+2+2 b)}{(a-k+2-2 b)(a-k-2 b)}-2(a-k)\right. \\
& \left.+(a-k+4 b) \frac{(a-k-4-2 b)(a-k-2-2 b)}{(a-k-2+2 b)(a-k+2 b)}\right) a_{1, k}=0 .
\end{aligned}
$$

By (3.11) and the discussion after (3.13), we know the coefficient of $a_{1, k}$ must be zero. We obtain $b=-1$ or $-\frac{1}{2}$. Note that the case of $b=-\frac{1}{2}, b^{\prime}=0$ is contained in Case 1. So we only need to consider the case of $b=-1, b^{\prime}=\frac{1}{2}$.

Let $j=1$ in (3.11), then $(a-k) a_{1, k}$ is a constant for all even $k$ or all odd $k$. We suppose that

$$
(a-k) a_{1, k}= \begin{cases}x_{0}, & k \text { is even } \\ x_{1}, & k \text { is odd }\end{cases}
$$

Let $i=j=1$ in (3.18), we can obtain that $x_{0}=x_{1}$. That is to say, $(a-k) a_{1, k}$ is a constant for all $k \in \mathbb{Z}$. If $a-k_{1}=0$ for some $k_{1} \in \mathbb{Z}$, then $a_{1, k}=\frac{a-k_{1}}{a-k} a_{1, k_{1}}=$ 0 for all $k \neq k_{1}$, a contradiction with (3.8). Thus $a-k \neq 0$ for all $k \in \mathbb{Z}$, i.e., $a \notin \mathbb{Z}$. Denote $(a-k) a_{1, k}^{ \pm}$by $d_{2}^{ \pm}$, where $d_{2}^{ \pm} \in \mathbb{C}$ are constants. By (3.6) , let $j=1$, we get

$$
\left(\frac{i}{2}-1\right) a_{i+1, k}=\left(a-(k+1)+\frac{i}{2}\right) a_{1, k}-(a-k-1) a_{1, k+1},
$$


then $a_{i, k}=a_{1, k}$ for all $i \in \mathbb{Z}, i \neq 3$. Again by (3.6) , let $j=2, i=1$, we have

$$
-\frac{3}{2} a_{3, k}=\left(a-(k+2)+\frac{1}{2}\right) a_{2, k}-(a-k-1) a_{2, k+1},
$$

then $a_{3, k}=a_{1, k}$. Therefore, $a_{i j}^{ \pm}=(a-j)^{-1} d_{2}^{ \pm}$for all $i, j \in \mathbb{Z}$, and by (3.10),

$$
d_{2}^{+} d_{2}^{-}=0, \quad\left(d_{2}^{+}\right)^{2}+\left(d_{2}^{-}\right)^{2} \neq 0 .
$$

Case 3. $b^{\prime}=-b-\frac{3}{2}$.

Following the arguments in Case 2 , we have $2 j a_{j, k}=2 j a_{-j, k-2 j}$, and

$$
\begin{gathered}
\left(a-k-j+i b^{\prime}\right) a_{j, k}+\left(a-k-j-2 i-i b^{\prime}\right) a_{j, k+2 i}=2(a-k-i-j) a_{j, k+i} \\
\left(\left(a-k-1+4 b^{\prime}\right) \frac{\left(a-k-3-2 b^{\prime}\right)\left(a-k-5-2 b^{\prime}\right)}{\left(a-k-1+2 b^{\prime}\right)\left(a-k-3+2 b^{\prime}\right)}-2(a-k-5)\right. \\
\left.+\left(a-k-9-4 b^{\prime}\right) \frac{\left(a-k-7+2 b^{\prime}\right)\left(a-k-5+2 b^{\prime}\right)}{\left(a-k-9-2 b^{\prime}\right)\left(a-k-7-2 b^{\prime}\right)}\right) a_{1, k+4}=0
\end{gathered}
$$

Then we obtain that $b=-\frac{3}{2}$ or -1 . The case of $b=-1\left(b^{\prime}=-\frac{1}{2}\right)$ is contained in Case 1. So we consider the case of $b=-\frac{3}{2}, b^{\prime}=0$. By (3.11), let $j=1$, we have

$$
(a-k-1) a_{1, k}=(a-k-3) a_{1, k+2},
$$

then $(a-k-1) a_{1, k}$ is a constant for all even $k$ or all odd $k$. By (3.19), similar to the argument in Case 2, we can obtain that $(a-k-1) a_{1, k}$ is a constant for all $k \in \mathbb{Z}$. If $a-k_{1}-1=0$ for some $k_{1}$, then $a_{1, k}=\frac{a-k_{1}-1}{a-k-1} a_{1, k_{1}}=0$ for all $k \neq k_{1}$. Also a contradiction with (3.8). Therefore, $a \notin \mathbb{Z}$. Now we denote $(a-k-1) a_{1, k}$ by $d_{3}$, i.e. $a_{1, k}=(a-k-1)^{-1} d_{3}$, for all $k \in \mathbb{Z}$. Let $j=1$ in (3.6) , then we have that

$$
\left(\frac{i}{2}-1\right) a_{i+1, k}=(a-k-1) a_{1, k}-\left(a-k-\frac{3}{2} i\right) a_{1, k+i} .
$$

Then

$$
a_{i, k}=a_{1, k+i-1}=(a-k-i)^{-1} d_{3} \text { for } i \neq 3 .
$$

Let $i=1, j=2$ in (3.6) , we get $-\frac{3}{2} a_{3, k}=(a-k-2) a_{2, k}-\left(a-k-\frac{3}{2}\right) a_{2, k+1}=-\frac{3}{2} a_{2, k+1}$, then $a_{3, k}=(a-k-3)^{-1} d_{3}$. Therefore, $a_{i j}^{ \pm}=(a-i-j)^{-1} d_{3}^{ \pm}$for all $i, j \in \mathbb{Z}$, and by (3.10),

$$
d_{3}^{+} d_{3}^{-}=0, \quad\left(d_{3}^{+}\right)^{2}+\left(d_{3}^{-}\right)^{2} \neq 0
$$

Case 4. $b^{\prime}=b-\frac{1}{2}$.

Note that if we act $\left(\frac{i}{2}-j\right) G_{i+j}^{ \pm}=\left[L_{i}, G_{j}^{ \pm}\right]$on $y_{k}$, we can obtain that

$$
\left(\frac{i}{2}-j\right) b_{i+j, k}^{ \pm}=(a-(k+j)+i b) b_{j, k}^{ \pm}-\left(a-k+i b^{\prime}\right) b_{j, i+k}^{ \pm}
$$

Similar to the discussion in case $1\left(b=b^{\prime}+\frac{1}{2}\right)$, we have $b_{i j}^{ \pm}=d_{1}^{\prime \pm}, i, j \in \mathbb{Z}$ for some $d_{1}^{\prime \pm} \in \mathbb{C}$, and by (3.10),

$$
d_{1}^{\prime+} d_{1}^{\prime-}=0, \quad\left(d_{1}^{\prime+}\right)^{2}+\left(d_{1}^{\prime-}\right)^{2} \neq 0 .
$$


Then it follows from (3.7) that

$$
a_{i j}^{ \pm}=2(a-k+2 i b)\left(d_{1}^{\prime \mp}\right)^{-1} .
$$

Until now, we get that

$$
a_{i j}^{ \pm}= \begin{cases}d_{1}^{ \pm}, & b^{\prime}=b+\frac{1}{2}, \\ (a-j)^{-1} d_{2}^{ \pm}, & b^{\prime}=\frac{1}{2}, \quad b=-1, \\ (a-i-j)^{-1} d_{3}^{ \pm}, & b^{\prime}=0, \quad b=-\frac{3}{2}, \\ 2(a-j+2 i b)\left(d_{1}^{\prime \mp}\right)^{-1}, & b^{\prime}=b-\frac{1}{2} .\end{cases}
$$

Again using (3.17), following the same arguments about $a_{i, j}^{ \pm}$, we have that

$$
b_{i j}^{ \pm}= \begin{cases}d_{1}^{\prime \pm}, & b=b^{\prime}+\frac{1}{2}, \\ (a-j)^{-1} d_{2}^{\prime \pm}, & b=\frac{1}{2}, \quad b^{\prime}=-1, \\ (a-i-j)^{-1} d_{3}^{\prime \pm}, & b=0, \quad b^{\prime}=-\frac{3}{2} \\ 2\left(a-j+2 i\left(b+\frac{1}{2}\right)\right)\left(d_{1}^{\mp}\right)^{-1}, & b=b^{\prime}-\frac{1}{2} .\end{cases}
$$

Obviously only the following two cases can occur:

$$
\begin{array}{lll}
a_{i j}^{ \pm}=d^{ \pm}, & b_{i j}^{ \pm}=2\left(a-j+2 i\left(b+\frac{1}{2}\right)\right)\left(d^{\mp}\right)^{-1}, & b^{\prime}=b+\frac{1}{2} . \\
b_{i j}^{ \pm}=d^{\prime \pm}, & a_{i j}^{ \pm}=2(a-j+2 i b)\left(d^{\prime \mp}\right)^{-1}, & b=b^{\prime}+\frac{1}{2},
\end{array}
$$

for some $d^{ \pm}, d^{\prime \pm} \in \mathbb{C}$. Together with (3.10), we obtain that

$$
\begin{aligned}
& \left(d^{+}\right)^{2}+\left(d^{-}\right)^{2}=0, \quad d^{+} d^{-}=0, \quad \text { and } \\
& \left(d^{\prime+}\right)^{2}+\left(d^{\prime-}\right)^{2}=0, \quad d^{\prime+} d^{\prime-}=0 .
\end{aligned}
$$

By rescaling basis $\left\{y_{i} \mid i \in \mathbb{Z}\right\}$ (or $\left\{x_{i} \mid i \in \mathbb{Z}\right\}$ ) if necessary, we can suppose $d^{ \pm}=1$ (or $\left.d^{\prime \pm}=1\right)$. Then we rewrite $(\underline{3.22})$ :

$$
\begin{array}{lll}
a_{i j}^{+}\left(\text {or } a_{i j}^{-}\right)=1, & b_{i j}^{-}\left(\text {or } b_{i j}^{+}\right)=2\left(a-j+2 i\left(b+\frac{1}{2}\right)\right), & b^{\prime}=b+\frac{1}{2} . \\
b_{i j}^{+}\left(\text {or } b_{i j}^{-}\right)=1, & a_{i j}^{-}\left(\text {or } a_{i j}^{+}\right)=2(a-j+2 i b), & b^{\prime}=b-\frac{1}{2} .
\end{array}
$$

Now we consider one of the cases of (3.23):

$$
a_{i j}^{+}=1, b_{i j}^{-}=2\left(a-j+2 i\left(b+\frac{1}{2}\right)\right), a_{i j}^{-}=b_{i j}^{+}=0, b^{\prime}=b+\frac{1}{2} .
$$

We want to determine the action of $H_{i}$ on $V$. Set $f_{00}=f, f_{00}^{\prime}=f^{\prime}$. Since $\left[H_{0}, G_{i}^{+}\right]=G_{i}^{+}$, we have that $f^{\prime}=f+1$. Similar to the arguments of [8], we get the following cases.

Case 5. $a-b, a-b^{\prime} \notin \mathbb{Z}$. We have some cases as follows:

$$
\begin{array}{llll}
\text { Subcase 5.1. } & f_{i j}=f, & f_{i j}^{\prime}=f+1 ; & b^{\prime}=b+\frac{1}{2} . \\
\text { Subcase 5.2. } & f_{i j}=\frac{a-j}{a-i-j} f, & f_{i j}^{\prime}=f+1 ; & b=0, b^{\prime}=\frac{1}{2} . \\
\text { Subcase 5.3. } & f_{i j}=\frac{a-i-j}{a-j} f, & f_{i j}^{\prime}=f+1 ; & b=-1, \quad b^{\prime}=-\frac{1}{2} . \\
\text { Subcase 5.4. } & f_{i j}=f, & f_{i j}^{\prime}=\frac{a-j}{a-i-j}(f+1) ; & b=-\frac{1}{2}, b^{\prime}=0 . \\
\text { Subcase 5.5. } & f_{i j}=f, & f_{i j}^{\prime}=\frac{a-i-j}{a-j}(f+1) ; & b=-\frac{3}{2}, b^{\prime}=-1 .
\end{array}
$$


Note that

$$
\left[H_{i}, G_{j}^{+}\right]=G_{i+j}^{+} .
$$

Acting it on $x_{k}$, we compare the coefficients on the two sides, then we get contradictions for Subcases 5.2-5.5. And in Subcase 5.1, follows $\left[H_{i}, G_{j}^{-}\right] \cdot y_{k}=-G_{i+j}^{-} \cdot y_{k}$, one can get that $f=-2 b-2$. Then we obtain a representation $R A_{a, b}$ of $\mathcal{L}$ with basis $\left\{x_{i}, y_{i} \mid i \in \mathbb{Z}\right\}$ and the actions:

$$
\begin{aligned}
R A_{a, b}: L_{i} x_{j} & =(a-j+i b) x_{i+j}, & L_{i} y_{j} & =\left(a-j+i\left(b+\frac{1}{2}\right)\right) y_{i+j}, \\
H_{i} x_{j} & =-(2 b+2) x_{i+j}, & H_{i} y_{j} & =-(2 b+1) y_{i+j}, \\
G_{i}^{-} x_{j} & =G_{i}^{+} y_{j}=0, & G_{i}^{+} x_{j} & =y_{i+j}, \quad G_{i}^{-} y_{j}=2(a+i-j+2 i b) x_{i+j},
\end{aligned}
$$

(together with $c x_{i}=c y_{i}=0$ for all $i, j \in \mathbb{Z}$ ).

Obviously:

(i) As Vir-modules, $V_{0} \cong A_{a, b}$ and $V_{1} \cong A_{a, b^{\prime}}$, where $b$ and $b^{\prime}$ have some relations.

(ii) For all $i \in \mathbb{Z}, H_{i}$ acts as constants on $V_{0}$ and $V_{1}$.

Case 6. $a-b \in \mathbb{Z}$, then $a-b^{\prime} \notin \mathbb{Z}$. Similar to the discussion in case 5 , we have the following subcases:

$$
\begin{aligned}
& \text { Subcase 6.1. } f_{i j}=0, \quad f_{i j}^{\prime}=1 ; \quad b=0, \quad b^{\prime}=\frac{1}{2} \text {. } \\
& \text { Subcase 6.2. } f_{i j}=0, \quad f_{i j}^{\prime}=1 ; \quad b=-1, \quad b^{\prime}=-\frac{1}{2} \text {. } \\
& \text { Subcase 6.3. } f_{i j}=f \neq 0, \quad f_{i j}^{\prime}=f+1 ; \quad b=-\frac{1}{2}, \quad b^{\prime}=0 . \\
& \text { Subcase 6.4. } f_{i j}=f \neq 0, \quad f_{i j}^{\prime}=\frac{a-i}{a-i-j}(f+1) ; \quad b=-\frac{1}{2}, \quad b^{\prime}=0 . \\
& \text { Subcase 6.5. } \quad f_{i j}=\frac{a-j}{a-i-j} f, \quad f_{i j}^{\prime}=f+1 ; \quad b=0, \quad b^{\prime}=\frac{1}{2} . \\
& \text { Subcase 6.6. } f_{i j}=\frac{a-i-j}{a-j} f, \quad f_{i j}^{\prime}=f+1 ; \quad b=-1, \quad b^{\prime}=-\frac{1}{2} \text {. } \\
& \text { Subcase 6.7. } f_{i j}=f, \quad f_{i j}^{\prime}=\frac{a-i-j}{a-j}(f+1) ; \quad b=-\frac{3}{2}, \quad b^{\prime}=-1 .
\end{aligned}
$$

Again by (3.24), we know that only Subcases 6.2 and 6.3 can occur. It is not difficult to see that they are contained in (3.25).

Case 7. $a-b^{\prime} \in \mathbb{Z}$, then $a-b \notin \mathbb{Z}$. Similar to the discussion of case 6 , we obtain that if $a_{i j}^{+}=1, b_{i j}^{-}=2\left(a-j+2 i\left(b+\frac{1}{2}\right)\right), a_{i j}^{-}=b_{i j}^{+}=0, b^{\prime}=b+\frac{1}{2}$, the module $V$ has the form of $R A_{a, b}$, for some $a, b \in \mathbb{C}$.

Similarly, we can write the other three cases of (3.23):

$$
\begin{array}{rlrl}
R B_{a, b}: L_{i} x_{j} & =(a-j+i b) x_{i+j}, L_{i} y_{j} & =\left(a-j+i\left(b+\frac{1}{2}\right)\right) y_{i+j}, \\
H_{i} x_{j} & =(2 b+2) x_{i+j}, & H_{i} y_{j} & =(2 b+1) y_{i+j}, \\
G_{i}^{+} x_{j} & =G_{i}^{-} y_{j}=0, \quad G_{i}^{-} x_{j}=y_{i+j}, \quad G_{i}^{+} y_{j}=2(a+i-j+2 i b) x_{i+j},
\end{array}
$$

when $a_{i j}^{-}=1, b_{i j}^{+}=2\left(a-j+2 i\left(b+\frac{1}{2}\right)\right), a_{i j}^{+}=b_{i j}^{-}=0, b^{\prime}=b+\frac{1}{2}$. 


$$
\begin{aligned}
R A_{a, b}^{\prime}: \quad L_{i} x_{j} & =(a-j+i b) x_{i+j}, L_{i} y_{j}=\left(a-j+i\left(b-\frac{1}{2}\right)\right) y_{i+j}, \\
H_{i} x_{j} & =-2 b x_{i+j}, \\
G_{i}^{+} x_{j} & =G_{i}^{-} y_{j}=0, \quad H_{i} y_{j}=-(2 b+1) y_{i+j}, \\
G_{i}^{+} y_{j} & =x_{i+j}, \quad G_{i}^{-} x_{j}=2(a-j+2 i b) y_{i+j},
\end{aligned}
$$

when $a_{i j}^{-}=2(a-j+2 i b), b_{i j}^{+}=1, a_{i j}^{+}=b_{i j}^{-}=0, b^{\prime}=b-\frac{1}{2}$.

$$
\begin{aligned}
R B_{a, b}^{\prime}: L_{i} x_{j} & =(a-j+i b) x_{i+j}, & L_{i} y_{j} & =\left(a-j+i\left(b-\frac{1}{2}\right)\right) y_{i+j}, \\
H_{i} x_{j} & =2 b x_{i+j}, & H_{i} y_{j} & =(2 b+1) y_{i+j}, \\
G_{i}^{-} x_{j} & =G_{i}^{+} y_{j}=0, & G_{i}^{-} y_{j} & =x_{i+j}, \quad G_{i}^{+} x_{j}=2(a-j+2 i b) y_{i+j},
\end{aligned}
$$

when $a_{i j}^{+}=2(a-j+2 i b), b_{i j}^{-}=1, a_{i j}^{-}=b_{i j}^{+}=0, b^{\prime}=b-\frac{1}{2}$.

It is not difficult to see that $R A_{a, b} \cong R A_{a, b+\frac{1}{2}}^{\prime}, R B_{a, b} \cong R B_{a, b+\frac{1}{2}}^{\prime}$.

§3.2 Now we consider all the possible deformations of the representations which defined in section 3.1.

Case 1. Suppose $V$ is an indecomposable module which has the same composition factors as those of $R A_{a, b}$ (in this case $R A_{a, b}$ is reducible). Let $V^{\prime}$ be a non-zero submodule of $V$.

Subcase 1.1. There exists $x_{i} \in V^{\prime}$ for some $i \in \mathbb{Z}$. By the sixth equation of (3.25), we obtain that $y_{j} \in V^{\prime}$ for all $j \in \mathbb{Z}$. By the last equation of (3.25),

$$
G_{i}^{-} y_{k-i}=2(a+i-k+i+2 i b) x_{k}=2(a-k+2(b+1) i) x_{k} .
$$

we see that $V^{\prime}$ is a proper submodule of $V$ if and only if $a=k_{0}, b=-1$ for some $k_{0} \in \mathbb{Z}$. In this case we can suppose $a=0, b=-1$. Then

$$
V^{\prime}=\operatorname{span}_{\mathbb{C}}\left\{x_{i}, y_{j} \mid i, j \in \mathbb{Z}, i \neq 0\right\}
$$

is a nontrivial irreducible submodule of $V$, with the following relations:

$$
\begin{array}{ll}
L_{i} x_{k}=-(i+k) x_{i+k}, & L_{i} y_{j}=-\left(\frac{i}{2}+j\right) y_{i+j}, \\
H_{i} x_{k}=0, & H_{i} y_{j}=y_{i+j}, \\
G_{i}^{-} x_{k}=G_{i}^{+} y_{j}=0, & G_{i}^{+} x_{k}=y_{i+k}, \quad G_{i}^{-} y_{j}=-2(i+j) x_{i+j},
\end{array}
$$

for all $j, k \in \mathbb{Z}$ and $k \neq 0$.

In order to determine all possible actions on $V$, we suppose that

$$
L_{i} x_{0}=l_{i} x_{i}, \quad H_{i} x_{0}=h_{i} x_{i}, G_{i}^{ \pm} x_{0}=g_{i}^{ \pm} y_{i} \quad \text { for all } i \in \mathbb{Z} .
$$

Act $\left[L_{i-1}, H_{1}\right]=-H_{i}$ on $x_{0}$, we can obtain that

$$
h_{i}=i h_{1} \text { for all } i \in \mathbb{Z} .
$$

Applying $\left[L_{i}, G_{j}^{-}\right]=\left(\frac{i}{2}-j\right) G_{i+j}^{-}$to $x_{0}$, we obtain that

$$
-\left(\frac{i}{2}+j\right) g_{j}^{-}=\left(\frac{i}{2}-j\right) g_{i+j}^{-} .
$$


Set $j=0$, we get that $-\frac{i}{2} g_{0}^{-}=\frac{i}{2} g_{i}^{-}$, i.e., $g_{i}^{-}=-g_{0}^{-}$for $i \in \mathbb{Z} \backslash\{0\}$. Set $j=-i$ in (3.32), we get that $-\left(\frac{i}{2}-i\right) g_{-i}^{-}=\left(\frac{i}{2}+i\right) g_{0}^{-}$, i.e., $g_{-i}^{-}=\frac{3}{2} g_{0}^{-}$for $i \in \mathbb{Z} \backslash\{0\}$. Then we must have that

$$
g_{i}^{-}=0 \text { for all } i \in \mathbb{Z} \text {. }
$$

Applying $\left[L_{i}, G_{j}^{+}\right]=\left(\frac{i}{2}-j\right) G_{i+j}^{+}$to $x_{0}$, we obtain that

$$
l_{i}=-\left(\frac{i}{2}+j\right) g_{j}^{+}-\left(\frac{i}{2}-j\right) g_{i+j}^{+} .
$$

Following $\left[H_{i}, G_{j}^{+}\right] \cdot x_{0}=G_{i+j}^{+} \cdot x_{0}$, we get that

$$
h_{i}=g_{j}^{+}-g_{i+j}^{+} \text {for all } i, j \in \mathbb{Z} .
$$

Set $j=0$, and by (3.31), one can get that

$$
g_{i}^{+}=g_{0}^{+}-i h_{1}
$$

It follows (3.34) that

$$
l_{i}=\frac{i^{2}}{2} h_{1}-i g_{0}^{+} .
$$

If $h_{1}=0$, then

$$
l_{i}=-i g_{0}^{+}, \quad h_{i}=0, \quad g_{i}^{-}=0, \quad g_{i}^{+}=g_{0}^{+},
$$

and it satisfies (3.30) (rescaling $x_{0}$ by $\left.\left(g_{0}^{+}\right)^{-1} x_{0}\right)$. Then it is not a deformation of $V$. Hence we suppose that $h_{1} \neq 0$. Rescaling $x_{0}$ by $h_{1}^{-1} x_{0}$, and follows (3.34), (3.36), we can obtain that

$$
l_{i}=-i \frac{g_{0}^{+}}{h_{1}}+\frac{i^{2}}{2}, \quad h_{i}=i, \quad g_{i}^{+}=\frac{g_{0}^{+}}{h_{1}}-i, \quad g_{i}^{-}=0 .
$$

Then we get a deformation of $R A_{a, b}$, denoted by $R A_{\alpha}$, which is an indecomposable module with the following relations:

$$
\begin{array}{rlrl}
R A_{\alpha}: & L_{i} x_{k}=-(i+k) x_{i+k}, & L_{i} x_{0}=\left(i \alpha+\frac{i^{2}}{2}\right) x_{i}, & L_{i} y_{j}=-\left(\frac{i}{2}+j\right) y_{i+j}, \\
H_{i} y_{j}=y_{i+j}, & H_{i} x_{k}=0, & H_{i} x_{0}=i x_{i}, \\
G_{i}^{-} x_{j}=G_{i}^{+} y_{j}=0, & G_{i}^{-} y_{j}=-2(i+j) x_{i+j}, & \\
G_{i}^{+} x_{k}=y_{i+k}, & G_{i}^{+} x_{0}=-(\alpha+i) y_{i}, &
\end{array}
$$

where $\alpha=-\frac{g_{0}^{+}}{h_{1}} \in \mathbb{C}, j, k \in \mathbb{Z}$ and $k \neq 0$.

Subcase 1.2. There exists $y_{j_{0}} \in V^{\prime}$ for some $j_{0} \in \mathbb{Z}$. By (3.25), in order to make $V^{\prime}$ is a proper submodule of $V$, we must have that for all $i \in \mathbb{Z}$,

$$
G_{i}^{-} y_{j_{0}}=2\left(a+i-j_{0}+2 i b\right) x_{i+j_{0}}=0
$$

it follows that $a=j_{0}, b=-\frac{1}{2}$. Without loss of generality, we can suppose that $j_{0}=0$, then we have $a=0, b=-\frac{1}{2}$. Therefore, $V^{\prime}=\mathbb{C} y_{0}$ is a trivial proper 
submodule of $V$, and set $V^{\prime \prime}=\operatorname{span}_{\mathbb{C}}\left\{x_{i}, y_{j} \mid i, j \in \mathbb{Z}, j \neq 0\right\}$. We have the following relations:

$$
\begin{array}{ll}
L_{i} x_{j}=-\left(\frac{i}{2}+j\right) x_{i+j}, & L_{i} y_{k}=-k y_{i+k}, \\
H_{i} x_{j}=-x_{i+j}, & H_{i} y_{k}=0, \\
G_{i}^{-} x_{k}=G_{i}^{+} y_{j}=0, & G_{i}^{+} x_{k}=y_{i+k}, \quad G_{i}^{-} y_{j}=-2 j x_{i+j},
\end{array}
$$

where $j, k \in \mathbb{Z}$ and $k \neq-i$. Suppose that

$$
L_{i} y_{-i}=l_{i} y_{0}, \quad H_{i} y_{-i}=h_{i} y_{0}, \quad G_{i}^{ \pm} x_{-i}=g_{i}^{ \pm} y_{0} .
$$

Similar to the arguments about Case 1, one can get that

$$
l_{i}=i g_{0}^{+}+\frac{i^{2}}{2} h_{1}, \quad g_{i}^{+}=g_{0}^{+}+i h_{1}, \quad g_{i}^{-}=0, \quad h_{i}=i h_{1} .
$$

If $h_{1}=0$, then

$$
l_{i}=i g_{0}^{+}, \quad g_{i}^{+}=g_{0}^{+}, \quad g_{i}^{-}=0, \quad h_{i}=0 .
$$

It is not a deformation too, so we suppose that $h_{1} \neq 0$. Rescaling $y_{0}$ by $h_{1} y_{0}$, we can get a new representation of $\mathcal{L}$, we denote it by $R A^{\beta}$.

$R A^{\beta}$ : satisfies (3.40) and the following relations $\left(\operatorname{set} \beta=\frac{g_{0}^{+}}{h_{1}}\right)$ :

$$
L_{i} y_{-i}=\left(i \beta+\frac{i^{2}}{2}\right) y_{0}, \quad H_{i} y_{-i}=i y_{0}, \quad G_{i}^{-} x_{-i}=0, \quad G_{i}^{+} x_{-i}=(\beta+i) y_{0} .
$$

Obviously, $R A_{\alpha}$ has a nontrivial submodule with codimention one, and $R A^{\beta}$ has a trivial submodule with dimention one.

Case 2. Now we discuss the deformations of $R B_{a, b}$. Since the discussion is similar to Case 1 , we will not give the detail, and only enumerate the results. $R B_{a, b}$ also has two deformations:

$$
\begin{array}{rlll}
R B_{\alpha}: & L_{i} x_{k}=-(i+k) x_{i+k}, & L_{i} x_{0}=-\left(i \alpha+\frac{i^{2}}{2}\right) x_{i}, & L_{i} y_{j}=-\left(\frac{i}{2}+j\right) y_{i+j}, \\
H_{i} y_{j}=-y_{i+j}, & H_{i} x_{k}=0, & H_{i} x_{0}=i x_{i}, \\
G_{i}^{+} x_{j}=G_{i}^{-} y_{j}=0, & G_{i}^{+} y_{j}=-2(i+j) x_{i+j}, & \\
G_{i}^{-} x_{k}=y_{i+k}, & G_{i}^{-} x_{0}=(\alpha+i) y_{i}, &
\end{array}
$$

where $\alpha=\frac{g_{0}^{-}}{h_{1}} \in \mathbb{C}, h_{1} \neq 0, j, k \in \mathbb{Z}$, and $k \neq 0$.

$$
\begin{array}{llll}
R B^{\beta}: & L_{i} x_{j}=-\left(\frac{i}{2}+j\right) x_{i+j}, & L_{i} y_{k}=-k y_{i+k}, & L_{i} y_{-i}=-\left(i \beta+\frac{i^{2}}{2}\right) y_{0}, \\
& H_{i} x_{j}=x_{i+j}, & H_{i} y_{k}=0, & H_{i} y_{-i}=i y_{0}, \\
& G_{i}^{+} x_{j}=G_{i}^{-} y_{j}=0, & G_{i}^{-} x_{k}=y_{i+k}, & \\
G_{i}^{-} x_{-i}=-(\beta+i) y_{0}, & G_{i}^{+} y_{j}=-2 j x_{i+j},
\end{array}
$$

where $\beta=-\frac{g_{0}^{-}}{h_{1}} \in \mathbb{C}, h_{1} \neq 0, j, k \in \mathbb{Z}$ and $k \neq-i$.

This completes the proof of Theorem 1.1. 


\section{References}

[1] M. Ademollo, L. Brink, A. d'Adda, R. Auria, E. Napolitano, S. Sciuto, E. del Giudice, P. di Vecchia, S. Ferrara, F. Gliozzi, R. Musto, R. Pettorino, Supersymmetric strings and colour confinement. Phys. Lett. B 62, 105(1976).

[2] E. Arbarello, C. De Concini, V.G. Kac and C. Procesi, Moduli spaces of curves and representation theory, Comm. Math. Phys., 117(1), 1-36(1988).

[3] S.L. Cheng, V.G. Kac, A new $N=6$ superconformal algebra. Comm. Math. Phys. 186, 219-231(1997).

[4] V.G. Kac, Lie superalgebras. Adv. Math. 26, 8-97(1977).

[5] V.G. Kac, J.W. van de Leuer, On classification of superconformal algebras. Strings 88, Sinapore: World Scientific, (1988).

[6] I. Kaplansky, L.J. Santharoubane, Harish-Chandra modules over the Virasoro algebras. in MSRI Publ.4, 217-231(1987).

[7] E. Kiritsis, Character formula and the structure of the represetations of the $N=$ 1, $N=2$ superconformal algebrass. Int. J. Mod. Phys. A 3, 1871-1906(1988).

[8] R. Lu, K. Zhao, Classification of Irreducible Weight Modules over the Twisted Heisenberg-Virasoro Algebras. Arxiv: math.ST/0510194 v1, 10 Oct 2005.

[9] C. Martin, A. Piard, Indecomposable modules for the Virasoro Lie algebra and a conjecture of Kac. Comm. Math. Phys. 137, 109-132(1991).

[10] Y. Su, Classification of Harish-Chandra Modules over the Super-Virasoro Algebras. Comm. Alg. 23(10), 3653-3675(1995).

[11] Y. Su, A classification of indecompable $s l_{2}(\mathbb{C})$-modules and a conjecture of Kac on irreducible modules over the Virasoro algebra. J. Algebra, 161(1), 33-46(1993).

[12] Y. Su, K. Zhao, Generalized Virasoro and super-Virasoro algebras and modules of the intermediate series. J. Algebra, 252(1), 1-19(2002). 